\title{
AiMT
}

Advances in Military Technology

Vol. 14, No. 2 (2019), pp. 307-319

ISSN 1802-2308, eISSN 2533-4123

DOI 10.3849/aimt.01330

\section{System of Systems Architecture for Generic Torpedo Defence System for Surface Ships}

\author{
G. Jomon*, J.V. Jojish and T. Santhanakrishnan \\ Naval Physical and Oceanographic Laboratory, Kochi, India
}

The manuscript was received on 25 April 2019 and was accepted after revision for publication on 8 November 2019.

\begin{abstract}
:
Protecting war ships from torpedo attack is considered as the most challenging task in Anti-Submarine Warfare. Torpedo Defence System (TDS) is an essential fitment on modern high value platforms. Timely detection and identification of a torpedo attack is the primary function of a TDS. Localisation and countermeasures against the weapon are the next stage in torpedo defence. Detection of modern torpedoes with long endurance can only be achieved using an underwater sensor suit with wide frequency coverage. Tactical use of multiple counter measures is essential for evading an attacking torpedo with intelligent homing mechanism. Individual sensors and counter measures are no longer considered as a solution; they are rather considered as components of a large network of systems to protect the platform from torpedo attack. A System of Systems (SOS) architecture with multiple sensors, processing techniques, countermeasures and tactics are presented in this paper for achieving fail-safe torpedo defence capability.
\end{abstract}

\section{Keywords:}

systems engineering, system of systems, requirement analysis, torpedo defence system

\section{Introduction}

'Torpedo' remains one of the oldest and deadliest weapons in naval warfare against both surface and submarine targets. Depending on the size, torpedoes are classified as heavy-weight and light-weight torpedoes. The older torpedoes use gyroscopic guidance for either straight running or pattern running. However, the modern torpedoes rely on acoustics for homing and attacking a target. Torpedo defence is a major area of concern for both surface ships and submarines. The defence against any underwater threat is mainly achieved through Anti-Submarine Warfare (ASW) operations, which is a complex series of operations executed by a surface platform with the aim of de-

\footnotetext{
* Corresponding author: Naval Physical and Oceanographic Laboratory, Thrikkakara P.O., Kochi-682021, Kerala, India.E-mail: jomong@npol.drdo.in
} 
tecting and neutralizing the hostile submarines before they launch the weapons, mainly torpedoes. In spite of the available advanced ASW systems, it should be taken into consideration that nowadays, in shallow waters, the hostile submarines are likely to succeed very often in launching their torpedoes before they are detected by the ASW units [1]. Hence, present day maritime warfare requires systems that can directly engage the attacking torpedo.

The two essential functionalities of a torpedo defence system are to detect the torpedo and to help the platform to evade its attack. There are two approaches for the latter task: one is hard-kill and the other is soft-kill. In hard-kill, the attacking torpedo is destroyed by another torpedo, which is an anti-torpedo torpedo. In soft-kill, the torpedo is lured away from the platform and its trajectory is controlled using decoys till its battery-life gets exhausted. The soft-kill method is generally done in a layered manner wherein the responsibility to defend the torpedo is passed on to different layers. Detection, tracking and classification of torpedo targets is the first step in torpedo defence. Identification of the torpedo threat parameter is the next step. Executing escape manoeuvre and tactical deployment of available countermeasures are the final steps in torpedo defence.

Torpedoes work either in passive, active or mixed modes. In active homing, the acquisition range is generally set by its ping rate. The general detection process is an energy detection and modern torpedoes do have correlators and FFT processing [2]. Passive homing is more effective against surface ships because surface ships create considerable noise due to propulsion. Passive homing torpedoes are more effective against surface targets, however, they are not effective against submerged diesel electric submarines. The torpedo homing frequencies are generally in the high frequency band because of the size limitations of the torpedo homing head. They use variable pulse widths and multiple transmission waveforms.

Torpedoes usually follow helical pattern search. Torpedoes are limited by their frequency of operation due to their small size as compared to the targets. Both active and passive homing ranges are restricted by their operating frequency. To compensate for these short ranges, wire guidance is used for extending the range. Modern torpedoes have two-way wire communication with the torpedo fire control system.

Torpedo attack is a covert operation. Modern heavy-weight torpedoes attack a ship either in autonomous or wire-guided mode. Torpedoes always run at higher speeds compared to ships or submarines. Protecting war ships from torpedo attack is considered as the most challenging task in Anti-Submarine Warfare. The main functions of a Torpedo Defence System (TDS) are to detect, localize and to take evasive and counter actions against an attacking torpedo [3]. Typical configuration of a torpedo defence system for surface ships is shown in Fig. 1.

System engineering practices are followed in developing defence systems. System Engineering is a robust approach to the design, creation, and operation of systems $[4,5]$. TDS is a complex system, which can be considered as a unique model of System of Systems (SOS). A system of systems is an arrangement of set of systems that are heterogeneous and independently operable on their own but are networked together for achieving unique capabilities. Both individual systems and SOS conform to the accepted definition of a system in which each consists of parts, relationships, and a whole that is greater than the sum of the parts [6]. SOS architecture is capable of achieving operational capability beyond the level on which the systems can provide it independently. This will demand more information sharing between systems and in- 
tern certain design modifications of systems. SOS architecture should include the following details:

- concept of operation: How the system is going to be operated by users during an operational scenario,

- system details including data flow and functional information,

- end to end functionality with communications protocols.

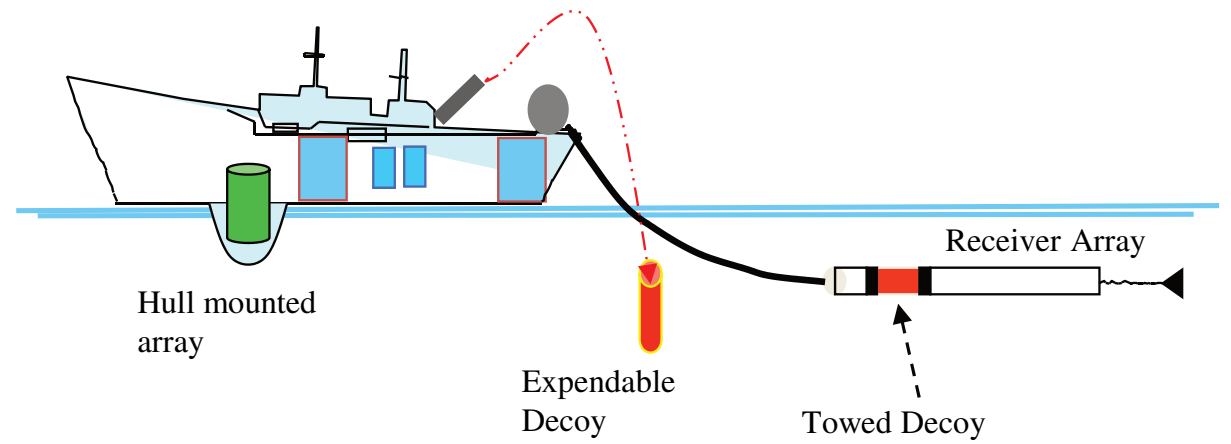

Fig. 1 Torpedo defence system configuration for surface ships

\section{Generic Torpedo Defence System}

TDS can be viewed as combination of complex systems working together cohesively to achieve the common goal of defending torpedo attack on a surface ship. Detection, classification, localisation and effective deployment of counter measures are the major objectives of TDS, as described in Fig. 2. A generic contemporary torpedo defence system comprises of five distinct elements:

- a set of acoustic sensors which detects an inbound torpedo threat,

- a highly automated target classifier to identify torpedo targets,

- target localization algorithm to localize torpedo targets,

- an automated information processor for threat evaluation, and tactical advice for evasive manoeuvring and deployment of countermeasures,

- a range of countermeasure solutions such as towed decoys and expendable decoys designed to lure the incoming torpedo away from its intended target (soft-kill) or destruction of the torpedo (hard-kill).

The technical challenges encountered when deploying such systems cannot be underplayed. Increasingly quiet propulsion systems mean that modern heavy-weight torpedoes can now approach their targets in 'near silent' mode; bearing ambiguity resolution is required to provide port/ starboard discrimination; a low false alarm rate is necessary to ensure operator's confidence and to conserve countermeasures expenditure; and countermeasures themselves must be of sufficient power and fidelity to ensure that they are effective against modern acoustic homing torpedoes.

At the moment, TDS systems are available for highly manoeuvrable small vessels having limited Detection Classification and Localisation (DCL) capability with too many false alarms and fairly effective soft-kill. Efforts are made to improve the DCL significantly and the effectiveness of the soft-kill. Parallel to that, hard-kill systems are under development, which are necessary for 'close-in defence' [7, 8]. One has to bear in mind that torpedo defence systems can only protect satisfactorily if an underwater 
sensor suite is used that has a full $360^{\circ}$ coverage and has got sufficiently long detection ranges under different sonic layer conditions. This means that hull-mounted sonar combined with a variable depth towed array will be required to meet various operating scenarios.

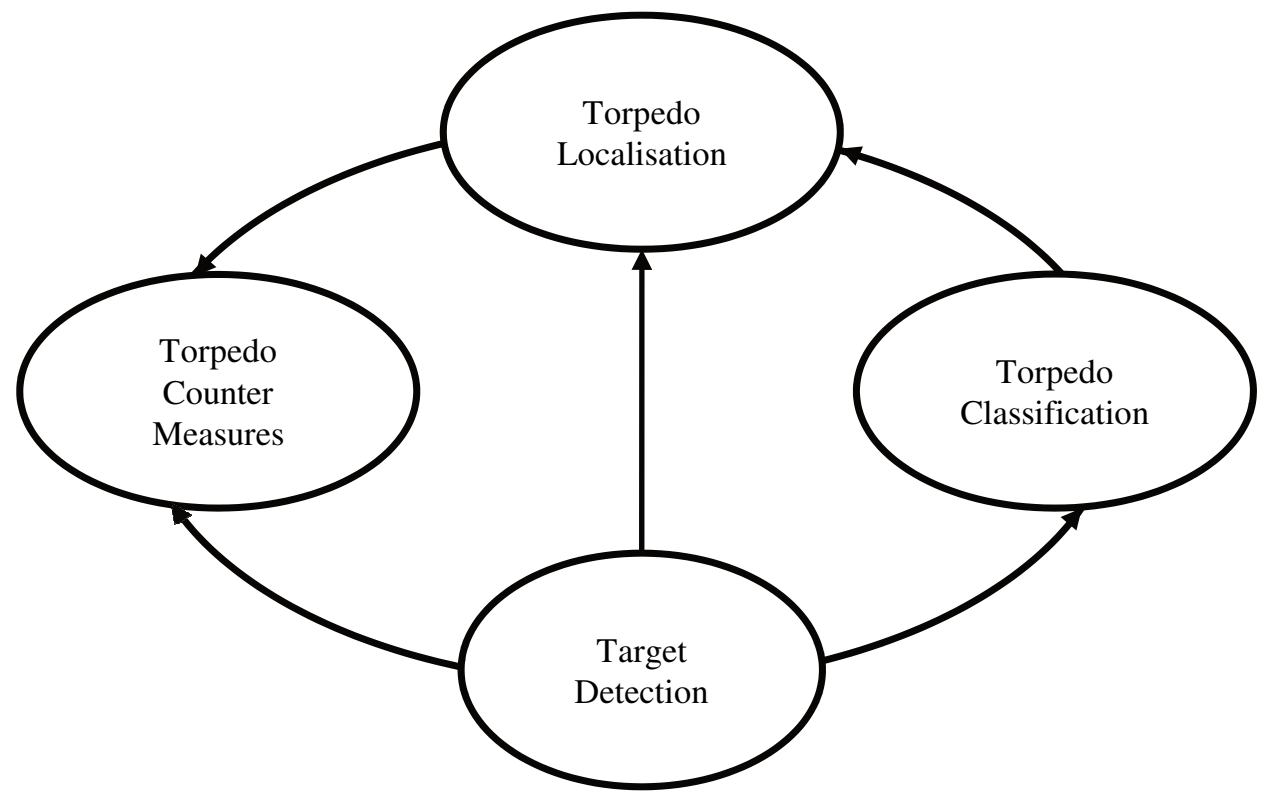

Fig. 2 Objectives of Torpedo Defence System

The following objectives are to be essentially met by the TDS:

- panoramic detection and tracking of all targets in the area of operation at maximum range,

- classification of torpedo targets at the earliest,

- localisation of torpedo targets using Target Motion Analysis (TMA) algorithms,

- threat analysis and recommendation of evasive manoeuvres and use of counter measures,

- effective use of countermeasures to evade torpedo attack.

\section{Torpedo Defence System: Concept of Operation}

Submarines are lethal because of certain specific features. One of the most important peculiarities of a submarine is stealth. The medium on which the submarine operates makes it a very discreet and elusive target. They are slow and have long endurance on periscope depth. However, they have limited endurance in submerged conditions due to battery power. Since they operate in a quiet environment, the sonar sensors have an additional advantage as compared to surface counterparts. They are vulnerable for attack by surface units or air units and submarines efforts shall always be made to avoid detection. They are lethal because they can very well fire a torpedo against a detected target. They also carry missiles which are typically used against a receding target.

To effectively detect a target, submarine uses a variety of sensors. The principal sensor is sonar which shall work in multiple modes. The most common mode is listen- 
ing to the radiated noise of the target by the passive sonar. The passive sonar has intercept modes also to detect transmissions if any. Following that submarine can use its active sonar, however, by operating it, the submarine compromises itself.

For obtaining the Fire Control Solution (FCS), the following sequence is carried out by the submarine. The target is first detected, and then classified. The target motion parameters are obtained by carrying out certain manoeuvers which will give the required parameters of the target, namely, bearing, range, course and speed. It will then select a suitable weapon, most probably a torpedo, and it will attack the target as per FCS recommendations. Thereafter, it has to get away to a safe location.

Torpedo defence systems typically follow the principle of layered defence which utilizes detection, classification, localization, workout escape tactics and deployment of countermeasures. The detection system is further divided into outer and inner layer defence with respect to the ranges of detection. TDS should be capable of detecting all the targets around the platform under all operating scenarios. Long-range detection including left-right ambiguity resolution can use a towed array sonar, whereas the hull-mounted sonar helps detect a torpedo at closer ranges and also from the head sector $[9,10]$.

Unlike conventional ASW where target is persistent, in torpedo systems, the target appears suddenly and is alive for very short time, either achieving its mission or failing. This demands that instead of operator detecting torpedo, the system has to detect it automatically. Robust torpedo classification algorithms with very low false alarm rate is necessary to ensure operator's confidence and to conserve countermeasures expenditure.

Torpedo detection is achieved typically using passive sonars. Passive sonars can provide only the target bearing information. Complex TMA algorithms are essential for localising the target from its bearing only information. Own ship manoeuvring is essential for computing target motion parameters. But, there are restrictions in own ship manoeuvring due to operational and safety reasons. Robust TMA algorithms are essential for calculating target parameters with minimum own ship manoeuvre. The TMA algorithms should accurately localise the attacking torpedo by computing its course, range and speed.

Threat analysis is another important requirement of a TDS. Threat analysis system assesses all possible threats in the vicinity and it assigns a threat priority level for all the targets [11]. The system will recommend a course to steer, as well as a countermeasure deployment to maximise escape probability. The system recommends possible escape manoeuvres and tactics to deploy expendable and towed decoys. Deployment of hard-kill systems also will be recommended by the system.

Once detected, the inner layer of defence is achieved by acoustic counter measures $[12,13]$. Towed acoustic decoy and expendable decoys are having a unique capability of seducing active and passive homing torpedoes. The towed acoustic decoy is a programmable decoy enabling the ship to transmit any type of signature including continuous wave, modulated waveforms, broad band noise, narrow band noise and any kind of amplitude modulation enabling deception of modern light and heavy-weight torpedoes. Also, the decoy carries intercept sensors, which can detect active transmissions from torpedo. The decoy can function in multiple modes, namely the echo repeater, broadband jammer and in autonomous mode. Hard-kill systems can also be used for evading a torpedo attack. Sequence of operations of a generic TDS system is described in Fig. 3. 


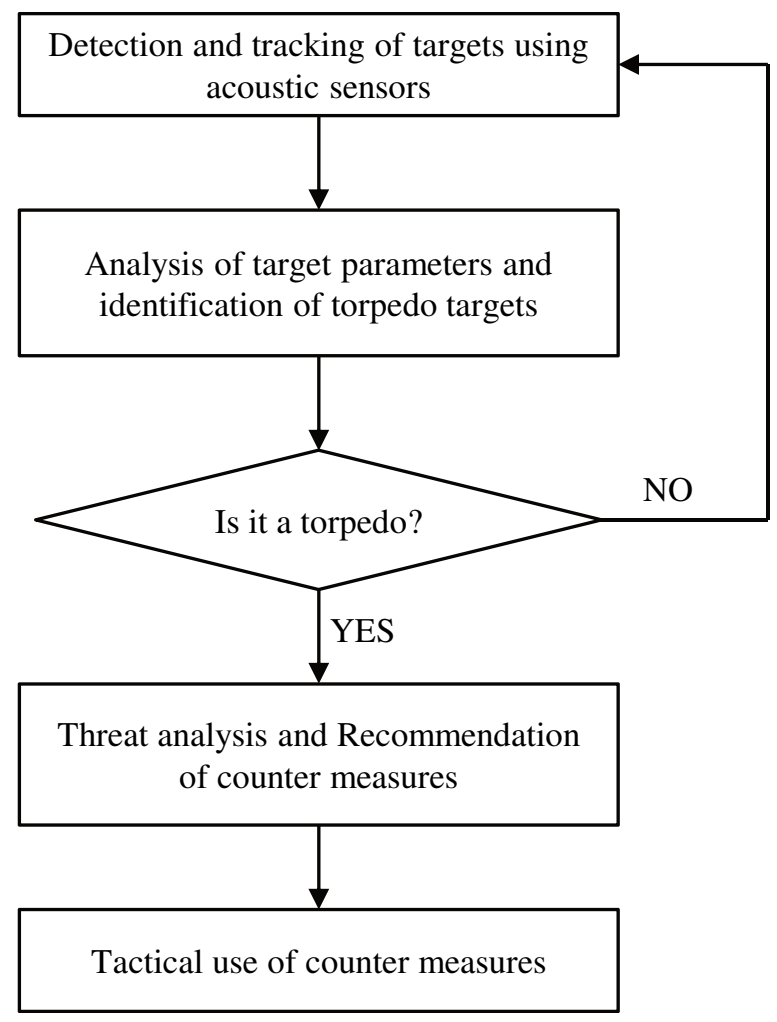

Fig. 3 TDS Sequence of operation

\section{Requirement Analysis}

Translating user requirements to system specifications is an essential stage during system design phase $[14,15]$. Many rounds of interactions and iterations are essential for capturing the user requirements. The mapping of high-level user requirements to system specifications forms the foundation for building the system capability. Systems engineering describes the system requirements as operational, functional and physical requirements [16].

\subsection{Operational Requirements}

The operational requirements of a TDS for surface ships shall specify the detailed operational specific requirements, such as basic mission requirements, performance needs, operational boundaries etc., as given in Tab. 1.

\subsection{Functional Requirements}

Detection, tracking, classification, localisation and decoying of an attacking torpedo are the major functions to be carried out by a TDS. These functions can be realised by selecting proper system configuration and by using signal processing algorithms. Functional requirements of TDS are given below:

- detection of targets using wideband acoustic sensors, 
- use of variable depth sensors to enhance detection probability,

- use of adaptive and advanced signal processing techniques for target detection [17],

- automatic target detection and tracking,

- robust torpedo classification algorithm using artificial intelligence and neural networks,

- multi-sensor data fusion and use of Kalman filter for target localization [18],

- simple and effective Human Machine Interface,

- use of multimode intelligent acoustic decoys for torpedo counter measures,

- destroying of attacking torpedo using anti-torpedo weapons.

Tab. 1 Operational requirements of TDS

\begin{tabular}{|c|c|c|}
\hline No. & Category & Requirements \\
\hline 1 & Mission Requirements & $\begin{array}{l}\text { - to detect, track, classify and locate active, passive } \\
\text { and wake homing torpedo attacks } \\
\text { - to decoy acoustic and non-acoustic homing torpe- } \\
\text { does } \\
\text { - to destroy an attacking torpedo using hard-kill } \\
\text { rockets } \\
\text { - locating and initiating counter attack on torpedo } \\
\text { firing platform }\end{array}$ \\
\hline 2 & $\begin{array}{l}\text { Performance } \\
\text { Requirements }\end{array}$ & $\begin{array}{l}\text { - long-range torpedo detection at firing range } \\
\text { - detection and localisation of torpedoes using pas- } \\
\text { sive, active or intercept sonars } \\
\text { - detection of targets at shallow water and deep water } \\
\text { and at different acoustic layers } \\
\text { - protecting ship from torpedo attack using decoys or } \\
\text { using hard-kill options }\end{array}$ \\
\hline 3 & Operational Needs & $\begin{array}{l}\text { - automatic operation with minimum human inter- } \\
\text { vention } \\
\text { - quick deployment and retrieval of the system } \\
\text { - high probability of detection and minimum false } \\
\text { alarm rate } \\
\text { - fail-safe system with adequate redundancy } \\
\text { - system should be able to fit on all major classes of } \\
\text { surface ships }\end{array}$ \\
\hline 4 & $\begin{array}{l}\text { Operational } \\
\text { Boundaries }\end{array}$ & $\begin{array}{l}\text { - target detection and tracking at own ship speed } \\
\text { upto } 22 \text { knots } \\
\text { - system should withstand escape speed of the plat- } \\
\text { form } \\
\text { - basic operations up to sea state } 4 \text { and system should } \\
\text { withstand all sea states }\end{array}$ \\
\hline
\end{tabular}

\subsection{Physical Requirements}

Physical configuration of TDS consists of wet end systems, launch systems, onboard electronics systems and counter measures and their launching systems as shown in Fig. 4. Wet end systems comprise of acoustic and non-acoustic sensors which are in direct 
contact with sea water. Launch systems are winch and handling systems for launch and retrieval of wet end systems. Onboard electronics houses the processing and display subsystems for processing sensor data and displaying the results. Acoustic decoys and anti-torpedo rockets are launched from pneumatically controlled launchers for defending torpedo attack.

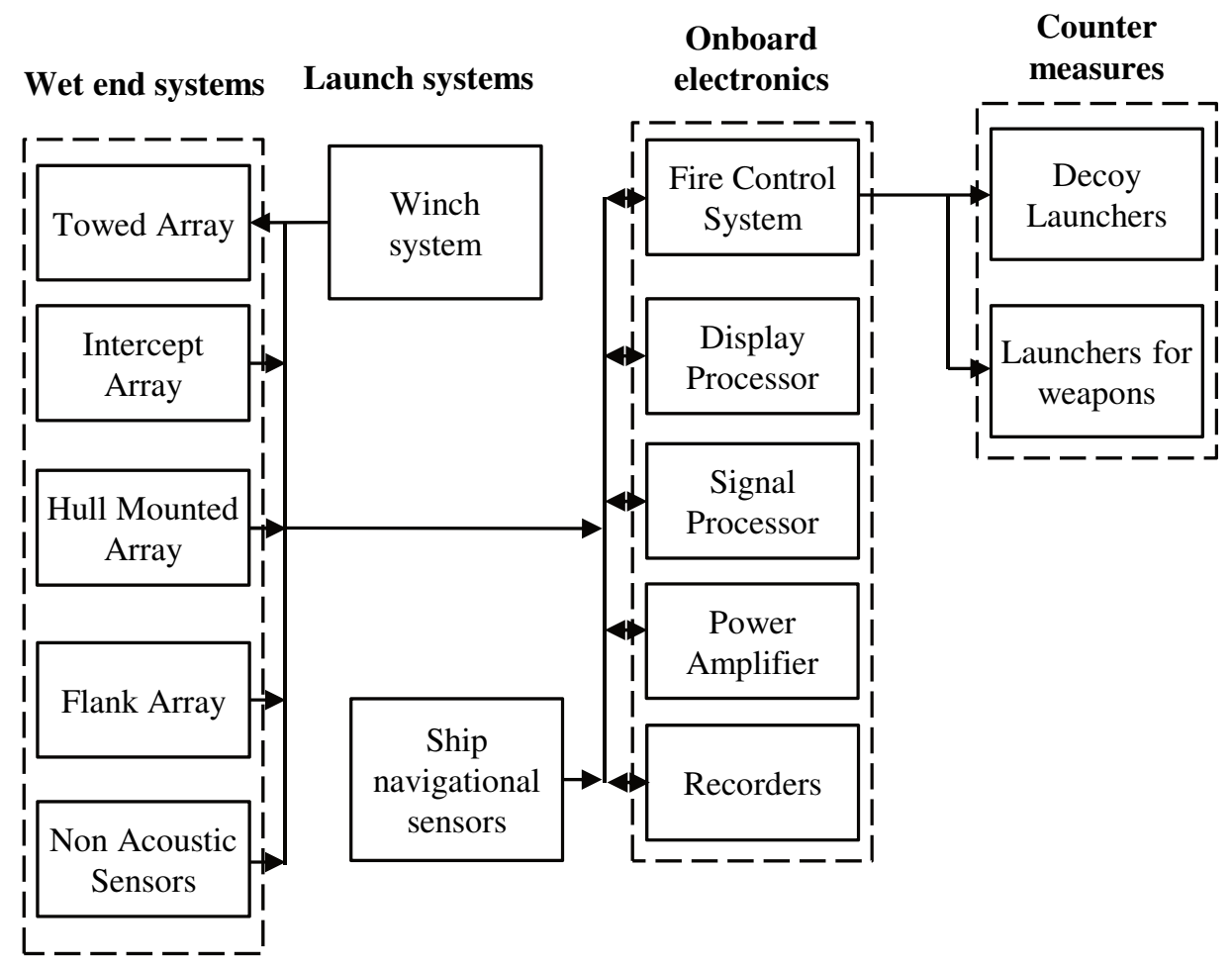

Fig. 4 Physical configuration of TDS

\section{SOS Architecture for TDS}

Defending torpedo attack is the most challenging task in underwater warfare. Independent systems working in isolation are not sufficient to defence torpedo threat. A consortium of sensors and countermeasure systems working with synergy under common command and guidance system is essential for achieving this most difficult task. A system of systems architecture for TDS is presented in Fig. 5. Major building blocks of TDS and their functional details are explained below.

\subsection{Acoustic and Non-acoustic Sensors for Torpedo Detection, Tracking and Clas- sification}

TDS essentially requires different types of sensors for effective torpedo detection under various operating conditions. The major considerations while selecting the sensors are its operating band, depth of operation and possibility of positioning it away from self-noise. The following five types of sensors are essentially used in TDS:

- hull-mounted array, 
- towed array,

- intercept array,

- flank array,

- non-acoustic sensors.

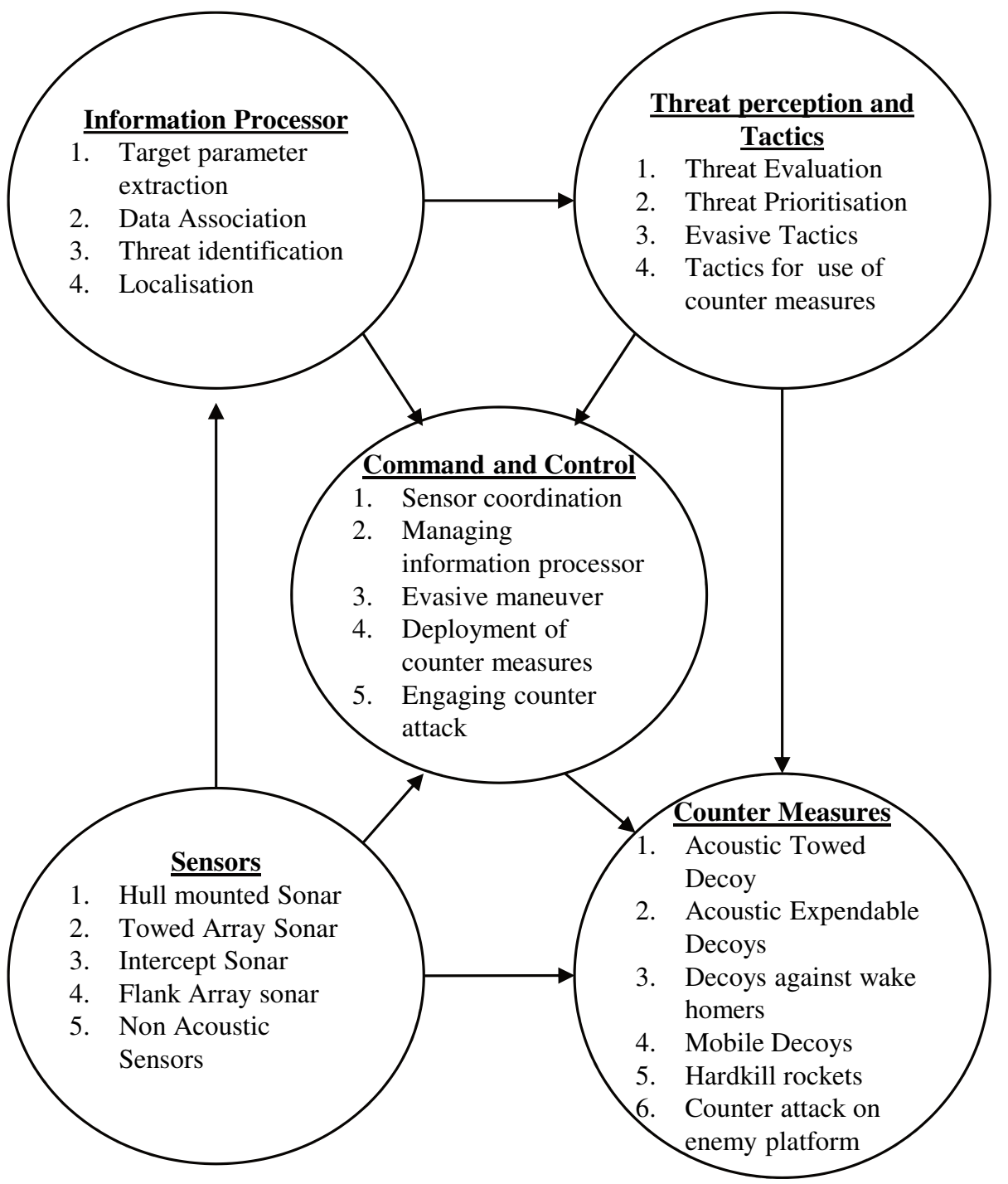

Fig. 5 SOS architecture for TDS

Identification of torpedo targets is an essential requirement of TDS. All countermeasure actions are initiated based on this classification. Target parameters extracted using different sensors are used for torpedo classifications. Bearings, frequencies, modulation frequencies are some of the features used for torpedo classification. Torpedo specific characteristics are different for different stages of torpedo run. Torpedo Confidence Level (TCL) is determined after analysing available information. Torpedo alarm is generated when TCL crosses set threshold. 


\subsection{Information Processor for Target Localization}

The TMA algorithm running on the Fire Control System (FCS) accurately computes the bearing, range and course of the torpedo at any instant using the target bearing derived from passive sonar sensors. Kalman filter based TMA algorithms is commonly used. Target parameters can also be derived using data association technique. The target observed bearings observed by different sensors located at different geometrical locations are used for computing target parameters.

\subsection{Tactics for Effective Torpedo Defence}

In a multi torpedo attacking scenario, threat evaluation and threat prioritization are the first step for torpedo defence. Recommendations for evasive manoeuvre and tactics for counter measure deployment are finalised based on the threat scenario. Evasive manoeuvre is finalised as per the torpedo counter attack tactics. Sequence of operation of counter measure devices and their modes of operation are finalised as part of torpedo defence tactics.

\subsection{Command and Control Unit}

Command and control system is the most important system in TDS. This unit coordinates activities of various systems in the SOS architecture. Command and Control Unit analyses the situation based on data available from various systems and take tactical decisions to maximise escape probability in a given scenario.

\subsection{Acoustic and Non-Acoustic Counter Measures}

List of countermeasure devices used as part of TDS are listed below:

- acoustic towed decoy,

- acoustic expendable decoys,

- mobile decoys,

- decoys against wake homers,

- hard-kill devices,

- counter-attack on enemy platform.

Acoustic homing torpedoes can be seduced and misguided using false acoustic sources called decoys $[19,20]$. Towed, expendable and mobile decoys are commonly used against both active and passive homing torpedoes. Wake-disturbing decoys are against wake-homing torpedoes. Anti-torpedo devices are used as explosives to destroy attacking torpedoes. The last stage in torpedo defence is attacking enemy launching platform through a counter attack to prevent further attacks.

\section{Data Flow and Communication}

SOS architecture of TDS is an intensive communication system. It follows distributed communication network architecture with a central command and control unit. Different systems in the SOS are working independently to achieve a common goal of torpedo defence. Command and Control Unit will coordinate between systems to achieve best results. Systems are communicating through Ethernet links using TCP/IP or UDP protocols. System to system interface protocol is clearly defined. Dataflow and communication diagram between systems are shown in Fig. 6.

Target bearing information received from sensors is used for torpedo identification and for computing torpedo motion parameters like course, range and speed. This 
information will be used by tactics processor for evaluating the threat and to workout escape tactics. Based on the recommendations of the tactics processor, Command and Control Unit will deploy suitable counter-measure to evade torpedo attack. Command and Control Unit is getting inputs directly from all the systems. If the normal data flow is affected due to malfunctioning of any of the participating systems, Command and Control Unit will get available data through alternate route. Standard communication protocols are proposed here to ensure future system growth by adding more systems and for smooth future upgradations.

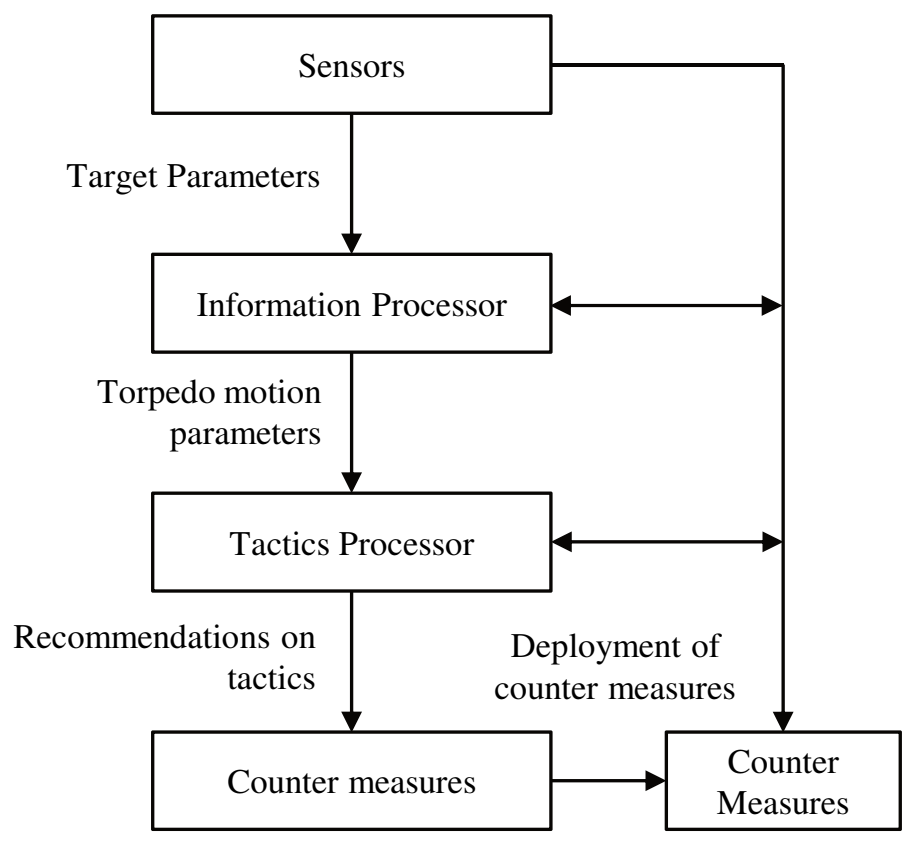

Fig. 6 Dataflow and communication diagram

\section{TDS with SOS Architecture vs. Contemporary Systems}

Defending torpedo attack on a surface ship is a complex task. Wide variety of sensors, countermeasures, information processing and decision making are involved in achieving this difficult task. Contemporary torpedo defence systems try to defend torpedo attack by deploying available set of sensors and counter measures. These systems are generally deployed for defending specific classes of torpedoes. These independent systems without proper centralised command and control system may not be sufficient to defend modern intelligent torpedoes. TDS with SOS architecture is a collection of task-oriented and dedicated systems that pool their resources and capabilities together to create a complex system which offers more functionalities and higher performance than simply a sum of the constituent systems. TDS with SOS architecture is having the capability to defend wide spectrum of torpedo threat. This system provides holistic view of the problem and provides solutions as per threat perception analysis. Centralised command and control system with distributed communication network is the backbone of SOS architecture. 


\section{Conclusion}

An SOS architecture for TDS system is presented in this paper. TDS system based on this architecture is capable of defending any potential torpedo attack on a surface platform. This system is capable of detecting and tracking of all types of torpedoes and decoying acoustic and wake homing torpedoes. TDS is a mission critical system. Robustness of the system is paramount. SOS architecture ensures redundancy for all critical functions. The uniquely distributed communication network with a central Command and Control Unit makes the system more robust against failures. A consortium of sensors and countermeasure systems working with synergy under common command and guidance system is the main advantage of SOS architecture. Coordination of geographically distributed and functionally independent systems for achieving the most difficult task with perfection is achievable though this architecture. TDS with SOS architecture is an essential fitment onboard all high-value platforms. Independent systems without centralised command and control system are not appropriate to defend surface ships against the threat from modern intelligent torpedoes.

\section{References}

[1] TOM, S.A. Strategic Antisubmarine Warfare and Naval Strategy. Lexington Books, USA, 1987. 390 p. ISBN 978-0-669-14015-6.

[2] RAMANARASAIAH, N.K. Introduction to Torpedo Technology. Defence Research \& Development Organisation, India, 1993. 93 p. ISBN 978-8-186-51400-9.

[3] KYUNG-MIN, S., HAE, S., JUNG, K. and TAG, G. Measurement of Effectiveness for an Anti-torpedo Combat System Using a Discrete Event Systems Specification-based Underwater Warfare Simulator. The Journal of Defense Modeling and Simulation, 2011, vol. 8, no. 3, p. 157-171. DOI 10.1177/ 1548512910390245.

[4] BLANCHARD, B.S. and FABRYCKY, W.J. Systems Engineering and Analysis. Pearson, USA, 2010. 800 p. ISBN 978-0-13-714843-1.

[5] INCOSE. Systems Engineering Handbook: A Guide for System Life Cycle Processes and Activities. Hoboken: Wiley, 2015. 304 p. ISBN 978-1-118-99940-0.

[6] JAMSHIDI, M. System of Systems Engineering: Innovations for the $21^{\text {st }}$ Century. Hoboken: Wiley, 2009. 616 p. ISBN 978-0-470-19590-1.

[7] MENG, J., WANG, Y.J., CAI, L. and WEI, R. Research on the Combination of Underwater Acoustic Countermeasure Equipments against Torpedo. In Proceedings of ICMES 2015, 2016, vol. 40, p. 1-3. DOI 10.1051.matecconf/ 20164002018.

[8] KWON, S.J., SEO, K.M., KIM, B. and KIM, T.G. Effectiveness Analysis of Anti-torpedo Warfare Simulation for Evaluating Mix Strategies of Decoys and Jammers. In Proceedings of Information and Communication Technology. Tokyo: Springer, 2012, vol. 4, p. 385-393. DOI 10.1007/978-4-431-54216-2_42.

[9] Technologies for Sonar Systems. Bulletin of Defence Research and Development Organisation, 2010, vol. 18, no. 4, p. 1-24. ISSN 0971-4413.

[10] WAITE, A.D. Sonar for Practising Engineers. Hoboken: Wiley, 2002, 256 p. ISBN 978-0-471-49750-9. 
[11] BHARATI, P., RAO, S.K. and KRISHNA, S.R. Generation and Analysis of Tactics for Anti-torpedo Defence System. In Proceedings of IEEE Conference on Information \& Communication Technologies. IEEE, 2013, p. 382-387. DOI 10.1109/CICT.2013.6558125.

[12] AKHIL, K.R., GHOSE, D. and RAO, K.S. Optimizing Deployment of Multiple Decoys to Enhance Ship Survivability. In Proceedings of 26th American Control Conference. IEEE, 2008, p. 1812-1817. DOI 10.1109/ACC.2008.4586755.

[13] NATHANIEL, J. and MARK, T. Initial Study on Expendable Acoustic Countermeasures for Torpedo Defence. DTA Report 341, 2012. ISSN 1175-6594.

[14]JIROTKA, M. and GOGUEN, J.A. Requirements Engineering: Social and Technical Issues. London: Academic Press, 1994, 296 p. ISBN 978-0-12-385335-6.

[15]MAIDEN, N. Scenarios for Acquiring and Validating Requirements. Automated Software Engineering, 1998, vol. 5, no. 4, p. 419-446. DOI 10.1023/A: 1008605412971.

[16] AJITHKUMAR, K., JOMON, G. and JAGATHYRAJ, V.P. Requirements Analysis of an Integrated Sonar Suite for Surface Ships: Systems Engineering Perspective. Defence Science Journal, 2014, vol. 64, no. 4, p. 366-370. DOI 10.14429/dsj.64.4486.

[17] GEORGE, A.D., GRACIA, J., KIM, K. and SINHA, P. Distributed Parallel Processing Techniques for Adaptive Sonar Beamforming. Journal of Computational Acoustics, 2002, vol. 10, no. 1, p.1-23. DOI 10.1142/S0218396X02000511.

[18] KALMAN, R.E. and BUSY, R.S. New Results in Linear Filtering and Prediction Theory. Journal of Basic Engineering, 1961, vol. 83, no. 1, p. 95-108. DOI 10.1115/1.3658902.

[19] CHEN, Y. and GIU, Y. Optimal Combination Strategy for Two Swim-Out Acoustic Decoys to Countermeasure Acoustic Homing Torpedo. In Proceedings of $4^{\text {th }}$ International Conference on Information Science and Control Engineering (ICISCE). IEEE, 2017, p. 1061-1065. DOI 10.1109/ICISCE.2017.222.

[20] CHEN, Y. and QIU, Y. Simulation-based Effectiveness Analysis of Acoustic Countermeasure for Ship Formation. In Proceedings of $4^{\text {th }}$ International Conference on Systems and Informatics (ICSAI). IEEE, 2017, p. 756-761. DOI 10.1109/ICSAI.2017.8248387. 